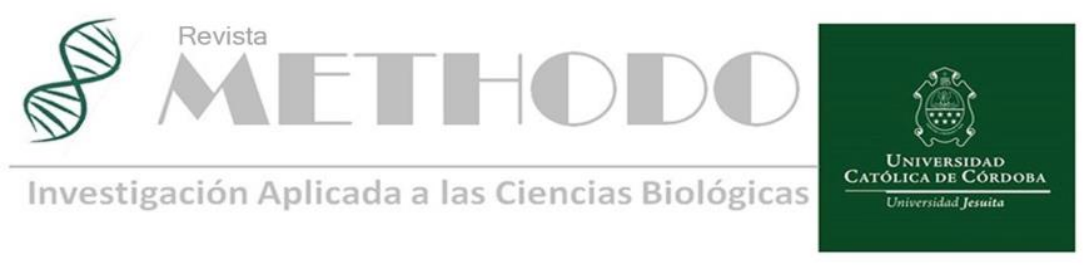

CASO CLINICO Rev. Methodo 2021;6(3):140-144 https://doi.org/10.22529/me.2021.6(3)07

Recibido 15 Ene. 20201| Aceptado 11 Abr. 2021|Publicado 02 Jul. 2021

\title{
Lesión hiperplasia de la encía: caso clínico, histológico y tratamiento. revisión de la literatura
}

\section{Hyperplastic lesion of the gingiva: clinical case, histological and treatment. Literature review}

\author{
María Eloísa Garay ${ }^{1}$ iD , Carolina Gonzalez ${ }^{1}$, Martin Brusa $^{2}$, Eduardo Piamonte ${ }^{2}$, René Panico², Ricardo \\ Crhistian Caciva ${ }^{2}$ \\ 1. Universidad Católica de Córdoba, Facultad de Ciencias de la Salud, Histología General y Dental, Escuela de Odontología \\ 2. Universidad Católica de Córdoba, Facultad de Ciencias de la Salud, Medicina Bucal, Escuela de Odontología \\ Correspondencia: Ricardo Caciva, E-mail: rccaciva@gmail.com
}

\section{Resumen}

Los épulis son lesiones bien conocidas que asientan en encía, de crecimiento generalmente lento; suelen aparecer como un tumor solitario localizado con base sésil o pediculada y color que varía de rojo, violáceo o rosa, según la vascularización de la lesión. Este crecimiento se considera de naturaleza no neoplásica (psuedotumor benigno hiperplásico), que se presenta en la cavidad oral en diversas formas clínicas e histológicas. El objetivo de este artículo es presentar un caso clínico de un épulis fibroso o hiperplasia gingival fibrosa circunscripta, su manejo clínico y la revisión de la literatura.

Palabras clave: Cavidad oral, encía, épulis, hiperplasia inflamatoria, etiopatogenia, tratamiento.

\begin{abstract}
Epulis are well-known gingival lesions, generally slow growing; they usually appear as a solitary localized tumor with a sessile or pedunculated base and a color that varies from red, purplish or pink, depending on the vascularity of the lesion. This growth is considered non-neoplastic in nature (benign hyperplastic psuedotumor), which occurs in the oral cavity in various clinical and histological forms. The objective of this article is to present a clinical case of a fibrous epulis or circumscribed fibrous gingival hyperplasia, its clinical management and a review of the literature.
\end{abstract}

Keywords: Oral cavity, gingiva, epulis, inflammatory hyperplasia, etiopathogenesis, treatment.

\section{Introducción}

El agrandamiento de los tejidos blandos de la cavidad oral a menudo presenta un desafío diagnóstico porque un grupo diversos de procesos patológicos pueden producir tales lesiones. Dentro de estos procesos patológicos se consideran hiperplasias reactivas, que se desarrollan en respuesta a una lesión tisular crónica y recurrente que estimula una respuesta de reparación tisular exuberante o excesiva. El épulis (epi = arriba o arriba y oulon = encía), es un término que se utiliza para describir el aspecto clínico de cualquier lesión que aparezca en la encía. Sin embargo, este término no da ninguna indicación sobre la 
naturaleza de la lesión ${ }^{1,2}$. El épulis es el pseudotumor benigno hiperplásico circunscrito más común de la encía, puede manifestarse semiológicamente como un tumor, pero es de naturaleza no neoplásica ${ }^{3}$. Es un tipo de hiperplasia que comúnmente se presenta en la cavidad bucal que histológicamente está constituida por una proliferación de tejido de granulación con infiltrado inflamatorio principalmente de linfocitos $^{4,5}$. Borguelli ${ }^{6}$ define clínicamente el épulis como un crecimiento anormal, circunscrito y de evolución crónica, localizado en la encía o cerca del reborde alveolar. Por otro lado, Donado ${ }^{7}$ considera el término épulis desde un punto de vista clínico y lo define como una tumoración inflamatoria crónica, granulomatosa, sobre la encía y en dependencia con el periostio o el periodonto.

A pesar de que los épulis no son de naturaleza neoplásica, algunos autores los clasifican dentro del grupo de los tumores benignos de la mucosa bucal ${ }^{6,8-10}$. Otros autores en cambio no están de acuerdo con esta consideración, prefieren catalogarlos como granulomas y, a su vez, diferenciarlos en subtipos según su histología ${ }^{11}$. Clínicamente, son lesiones pseudotumorales con características diversas. La etio-fisio-patogenia ha sido asociada a varios factores etiológicos como trauma, irritación crónica, fármacos, hormonales, impactación de alimentos, infecciones a biofilm/calculo dentro de la hendidura gingival y

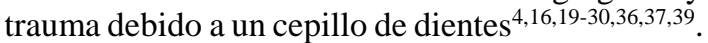
Generalmente, las lesiones hiperplásicas bucales siguen una evolución común en su formación. En primer lugar, se trata de una masa de tejido de granulación inflamado de consistencia blanda de color rojo intenso. Posteriormente, si el factor causal se mantiene, el tejido se vuelve más fibroso con el tiempo ${ }^{10,12}$. Existen diferencias en función de la localización, consistencia y relación con los tejidos circundantes. Para Anneroth y Sigurdson ${ }^{2}$, el verdadero "épulis" es el de células gigantes, ya que es el único que aparece exclusivamente en la encía y en el hueso alveolar. La clasificación que a continuación se propone, sigue el criterio histopatológico $2,6,39,41$ : a) Hiperplasia granulomatosa que incluye el épulis del embarazo, el granuloma piogénico, el épulis angiomatoso, el épulis telangiectásico ${ }^{13}$; b) Hiperplasia fibrosa ${ }^{8,14} \mathrm{y}$ c) Hiperplasia de células gigantes ${ }^{13}$.

Epidemiológicamente algunos autores observan una mayor frecuencia en el sexo femenino $1: 1,{ }^{56-}$ 9,15-18,41,42. El diagnostico diferencial incluye: fibroma osificante periférico, fibroma, fibroma odontogénico periférico, hemangioma, tejido de granulación convencional, inflamación gingival hiperplásica, sarcoma de Kaposi, angiomatosis bacilar, cáncer metastásico, angiosarcoma y linfoma no Hodgkin ${ }^{12,19,31-34}$.

\section{Caso clínico}

Paciente femenina de 64 años de edad que acudió a consulta odontológica al Servicio de Estomatología de la Escuela de Odontología, de la Facultad de ciencias de la Salud de la Universidad Católica de Córdoba, por presentar lesiones asintomáticas en encía de 1 año de evolución. Realizó enjuagues con $\mathrm{H} 2 \mathrm{O} 2$ + bicarbonato de $\mathrm{Na}$ y con colutorio "PLAX" sin mejoría, por lo que decidió realizar la consulta un especialista en Estomatología. En los antecedentes personales patológicos los datos de relevancia son Hipertensión arterial (HTA), medicada con Amlodipina $5 \mathrm{mg}$, Diurex 2,5 mg; Carvedilol 25 mg; Alplax 0,5 mg. Dieta Hiposódica y Diabetes Tipo II. Medicada Metformina $500 \mathrm{mg}$. Respecto a los hábitos, la paciente es fumadora pasiva hace 30 años, es bebedora social, consume vino tinto y toma 1 litro de mate a temperatura elevada diariamente. Al examen clínico presentaba, en encía vestibular (libre e insertada) de los incisivos central y lateral y en el canino del lado izquierdo, lesiones de aspecto tumoral de base sésil de superficie lisa, brillante del mismo color de la mucosa, de crecimiento lento, indoloro y con episodios de gingivorragia provocadas al trauma mecánico excesivo del cepillado (Figura.1), las mismas estaban en relación a la desadaptación protética fija como asociada a la enfermedad periodontal existente. Se solicitan interconsulta con médico tratante, como estudios complementarios de diagnóstico, tales como análisis de rutina (hemograma completo/glucemia) siendo sus valores normales y diagnósticos por imágenes (periapicales y radiografía panorámica), donde no hubo evidencia de reabsorción ósea asociada con la lesión.

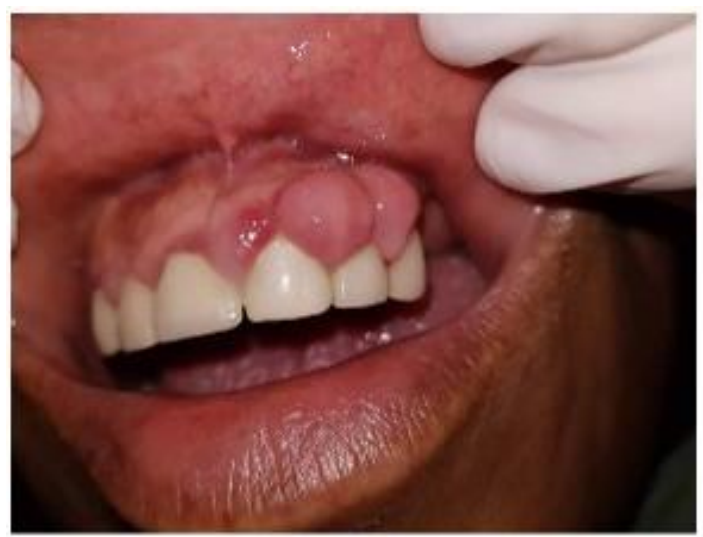

Figura1. Lesión de aspecto tumoral abarcando encía libre y parte de encía insertada en maxilar superior.

Se planteó posible diagnostico presuntivo como sus respectivos diagnósticos diferenciales nombrado con anterioridad. Se realizó biopsia excisional de las lesiones, seguida de alisado radicular de los dientes involucrados para la eliminación de los agentes causales

Revista Methodo: Investigación Aplicada a las Ciencias Biológicas. Universidad Católica de Córdoba. Jacinto Ríos 571 Bo Gral. Paz. X5004FXS. Córdoba. Argentina. Tel.: (54) 3514517299 / Correo: methodo@ucc.edu.ar / Web: methodo.ucc.edu.ar | CASO CLINICO Rev. Methodo 2021;6(3):140-144. 
como el cálculo/tártaro dental (Figura.2 y 3), se fijó en formol al $10 \%$ y se hicieron los estudios anatomopatológicos correspondientes para la confirmación diagnostica de: Épulis fibroso o hiperplasia gingival fibrosa circunscripta (Figura. 4 y 5 ).
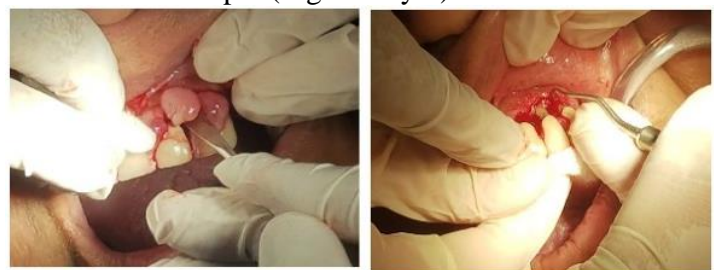

Figura 2 y 3. Biopsia por escisión y alisado radicular (destartraje).

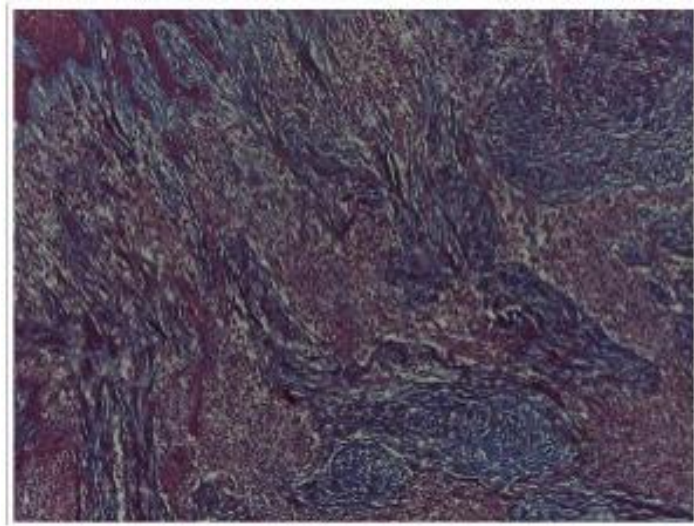

Figura 4. 10x. Tinción tricromico de masson, lesión constituida por tejido conectivo con abundantes haces irregulares de fibras de colágeno.

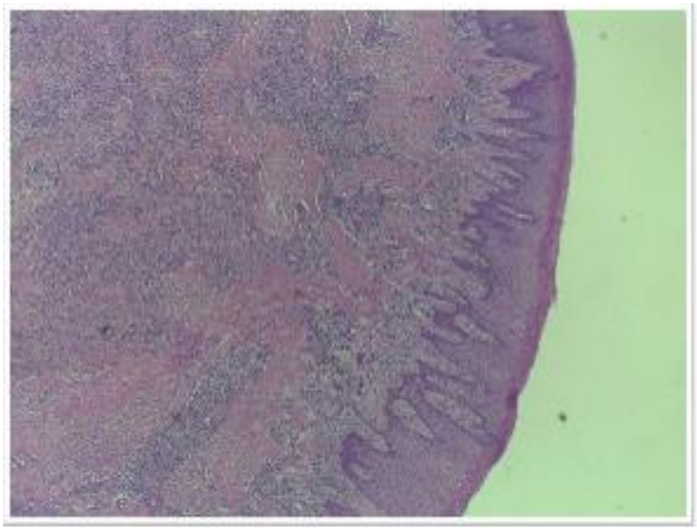

Figura 5. 10x. H/E se destaca un intenso infiltrado inflamatorio mononuclear con numerosas células plasmáticas. y se reconoce que la lesión esta revestida en superficie por epitelio plano estratificado, que presenta acantosis con elongación irregular de las redes de cresta.

\section{Discusión}

El épulis es una hiperplasia inflamatoria reactiva de naturaleza no neoplásica. El examen clínico exhaustivo así como el estudio histopatológico deben realizarse correctamente a fin de descartar enfermedades de mal pronóstico en vista de neoplasias primarias o metastásicas, entre otras, que pueden presentarse como épulis.

Actualmente se acepta universalmente que esta lesión es una reacción localizada exagerada del tejido conectivo en respuesta a una lesión menor o cualquier irritación subyacente (cálculos, mala higiene bucal, etc. $)^{35-37,40}$.

Toda lesión hiperplásica reactiva gingival debe tratarse eliminando el factor etiológico y extirpando la lesión. La escisión quirúrgica total es el tratamiento de elección para minimizar la recurrencia de la lesión. Además, es muy importante en el tratamiento preservar y mejorar el complejo muco-gingival dado que, si se realiza una tartrectomía y un raspado-alisado y pulido radicular pero no se mantiene un correcto estado periodontal estas lesiones poseen una alta probabilidad de recidiva ${ }^{38,40,42}$.

\section{Bibliografía}

1. Macleod RI, Soames JV. Epulides: a clinicopathological study of a series of 200 consecutive lesions. Br Dent J 1997; 163: 513. https://doi.org/10.1038/sj.bdj.4806194.

2. Anneroth G, Sigurdson A. Hyperplastic lesion of the gingiva and oral mucosa: a study of 175 cases. Acta Odontol Scand 1983; 41: 75-86. https://doi.org/10.3109/00016358309162306.

3. Gomes SR, Shakir QJ, Thaker PV y Tavadia JK, "Granuloma piógeno de la encía: ¿un nombre inapropiado? Reporte de un caso y revisión de la literatura" Revista de la Sociedad India de Periodoncia, vol. 17, no. 4, págs. 514-519, 2013

4. Jafarzadeh H, Sanatkhani M, Mohtasham N. Oral pyogenic granuloma: A review. J Oral Sci2006; 48:16775. https://doi.org/10.2334/josnusd.48.167.

5. Yao T, Nagai E, Utsunomiya T, Tsuneyoshi M. An intestinal counterpart of pyogenic granuloma of the skin. A newly proposed entity. Am J Surg Pathol 1995; 19:1054-60. DOI: 10.1097/00000478-199509000-00009.

6. Ceballos A. Medicina Bucal. Granada: Gráficas Anel; 1993. p. 120-41.

7. Donado M. Introducción a las lesiones tumorales bucomaxilofaciales. In: Donado M, ed. Cirugía Bucal. Patología y Técnica. Madrid: Masson; 1998. p. 559-63.

8. Ceballos A. Tumores benignos de la mucosa oral. En: Bagán JV, Ceballos A, Bermejo A, Aguirre JM, Peñarrocha M, eds. Medicina Oral. Barcelona: Masson; 1995. p. 177-85. 
9. Bhaskar SN. Patología bucal. Buenos Aires: El Ateneo; 1984. p. 343-91. 7. Blanco A, Blanco J, Suárez M, Álvarez N, Gándara JM. Hiperplasias inflamatorias de la cavidad oral. Estudio clínico e histológico de cien casos (I). Características generales. Av Odontoestomatol 1999; 15:553-61.

10. Blanco A, Blanco J, Suárez M, Álvarez N, Gándara JM. Hiperplasias inflamatorias de la cavidad oral. Estudio clínico e histológico de cien casos (I). Características generales. Av Odontoestomatol 1999; 15:553-61.

11. Margiotta V, Franco V, Giulana G. Epulide: aspetti istopatologici ed epidemiologici. Minerva Stomatol 1991; 40:51-5

12. Wood NK, Goaz PW. Lesiones exofíticas orales periféricas. En: Wood NK, Goaz PW, eds. Diagnóstico diferencial de las lesiones orales y maxilofaciales. Madrid: Harcourt Brace; 1998. p. 130-61.

13. Tumini V, Di Placido G, D’Archivio D, Del Giglio AM. Lesioni gengivali iperplastiche in gravidanza. Nota I. Epidemiologia, patologia e clinica. Minerva Stomatol 1998; 47:159-67.

14. Blanco A, Suárez M, Blanco J, Álvarez N, Gándara JM. Hiperplasias inflamatorias de la cavidad oral. Estudio clínico e histológico de cien casos (II). Características específicas de cada lesión. Av Odontoestomatol 1999;15: 563-76.

15. Rosa CG, Lay AC, Torre AC. Oral pyogenic granuloma diagnosis and treatment: A series of cases. Rev Odontol Mex 2017; 21:244 52.

16. Ojanotko-Harri AO, Harri MP, Hurttia HM, Sewón LA. Altered tissue metabolism of progesterone in pregnancy gingivitis and granuloma. J Clin Periodontol 1991; 18:2626.

17. Leal RM, Rodrigues AM, Mendonça AA, Chrcanovic BR. Oral pyogenic granuloma: Epidemiology of 171 cases. Rev Min Estomatol. 2004; 1:13-9. [Google Scholar]

18. Gordón-Núñez MA, de Vasconcelos Carvalho M, Benevenuto TG, Lopes MF, Silva LM, Galvão HC. Oral pyogenic granuloma: A retrospective analysis of 293 cases in a Brazilian population. J Oral Maxillofac Surg. 2010; 68:2185-8. [PubMed] [Google Scholar]

19. Regezi JA, Sciubba JJ, Jordan RC. Oral pathology: Clinical pathologic considerations. 4th ed. Philadelphia: WB Saunders; 2003. p. 115-6.
20. Murata M, Hara K, Saku T. Dynamic distribution of basic fibroblast growth factor during epulis formation: An immunohistochemical study in an enhanced healing process of the gingiva. J Oral Pathol Med 1997; 26:224-32.

21. Ainamo J. The effect of habitual tooth cleaning on the occurrence of periodontal disease and dental caries. Suom Hammaslaak Toim 1971; 67:63.

22. Neville BW, Damm DD, Allen CM, Bouquot JE. Oral and Maxillofacial Surgery. 2nd ed. Philadelphia: Saunders; 2002. p. 447-9.

23. Mussalli NG, Hopps RM, Johnson NW. Oral pyogenic granuloma as a complication of pregnancy and the use of hormonal contraceptives. Int J Gynaecol Obstet 1976; 14:187-91.

24. Bhaskar SN, Jacoway JR. Pyogenic granulom: clinical features, incidence, histology, and result of treatment: report of 242 cases. J Oral Surg 1966; 24(5): 391-8.

25. Atarbashi-Moghadam F, AtarbashiMoghadam S, Namdari M, ShahrabiFarahani S. Reactive Oral lesions associated with a dental implants: a sistematic review. J Investig Clin Dent. 2018; 10:1-7.

26. Amadei SU, Pereira AC, Silveira VA, Carmo ED, Scherma AP, Rosa LE. Prevalence of non-neoplastic proliferative processes in the oral cavity: A forty-year retrospective study. Dentistry clinic and research-UNITAU. 2009; 1:38-42. [Google Scholar]

27. China AL, Souza NM, Amanajás TA, Pedreira EN. Pyogenic granuloma: Reporting of atypical case on lower lip. Rev Para Med. 2010;24:(3/4). [Google Scholar]

28. Rossa C, Cartagena A, Torre A. Oral pyogenic granuloma diagnosis and treatment: A series of cases. Rev Odontol Mex. 2017; 21:244-52. [Google Scholar]

29. Shafer WG, Hine MK, Levy BM. Shafer's Textbook of Oral Pathology. 5th ed. Amsterdam: Elsevier Health Sciences; 2006. p. $459-61$.

30. Hosseini FH, Tirgari F, Shaigan S. Immunohistochemical analysis of estrogen and progesterone receptor expression in gingival lesions. Iran J Public Health 2006; 35:38 41 .

31. Eversole LR. Clinical Outline of Oral Pathology: Diagnosis and Treatment. 3rd ed. Hamilton: BC Decker; 2002. p. 113-4. 
32. Calonje E, Wilson-Jones E. Vascular tumors: Tumors and tumor like conditions of blood vessels and lymphatics. In: Elder D, Elenitsas R, Jaworsky C, Johnson B Jr, editors. Lever's Histopatology of the Skin. 8th ed. Philadephia: Lippicott-Raven; 1997. p. 895.

33. Raut A, Huryn J, Pollack A, Zlotolow I. Unusual gingival presentation of posttransplantation lymphoproliferative disorder: A case report and review of the literature. Oral Surg Oral Med Oral Pathol Oral Radiol Endod 2000; 90:436-41.

34. Steelman R, Holmes D. Pregnancy tumor in a 16-year-old: Case report and treatment considerations. J Clin Pediatr Dent 1992; $16: 217-8$.

35. Mathur LK, Bhalodi AP, Manohar B, Bhatia A, et al. Focal fibrous hyperplasia: a case report. Int J Dent Clin 2010; 2(4): 56-7.

36. Sangle VA, Pooja VK, Holani A, Shah N, et al. Reactive hyperplastic lesions of the oral cavity: A retrospective survey study and literature review. Indian J Dent Res. 2018 JanFeb;29(1):61-66. doi: 10.4103/ijdr.IJDR_599_16. PMID: 29442089.

37. Dutra KL, Longo L, Grando LJ, Rivero ERC. Incidence of reactive hyperplastic lesions in the oral cavity: a 10 year retrospective study in Santa Catarina, Brazil. Braz J Otorhinolaryngol. 2019 jul-Aug;85(4):399407. doi: 10.1016/j.bjorl.2018.03.006. Epub 2018 Apr 17. PMID: 29705120.

38. Zhu Y, Zhang H, Li C. The clinical application of partial removal periodontal surgery in the therapy of epulis. Medicine (Baltimore). 2019 jul;98(27): e16107. doi: 10.1097/MD.0000000000016107. PMID: 31277111 ; PMCID: PMC6635175.
39. Hunasgi S, Koneru A, Vanishree M, Manvikar V. Evaluación de las lesiones gingivales reactivas de la cavidad oral: un estudio histopatológico. J Oral Maxillofac Pathol. 2017 enero-abril; 21 (1): 180. doi: 10.4103 / jomfp. JOMFP_23_16. PMID: 28479713; PMCID: PMC5406807.

40. Kadeh H, Saravani S, Tayik M. Lesiones hiperplásicas reactivas de la cavidad oral. Irán J Otorhinolaryngol. Marzo de 2015; 27 (79): 137-44. PMID: 25938085; PMCID: PMC4409958.

41. Maturana-Ramírez A, Adorno-Farías D, Reyes-Rojas M, Farías-Vergara M, et al. A retrospective analysis of reactive hyperplastic lesions of the oral cavity: study of 1149 cases diagnosed between 2000 and 2011, Chile. Acta Odontol Latinoam. 2015;28(2):103-7. doi: $\quad 10.1590 /$ S1852-48342015000200002. PMID: 26355878.

42. Vidyanath S, Shameena PM, Johns DA, Shivashankar VY, et al. Lesiones hiperplásicas reactivas de la cavidad oral: una encuesta de 295 casos en una institución terciaria de salud en Kerala. J Oral Maxillofac Pathol. Septiembre-diciembre de 2015; 19 (3): 330-4. doi: 10.4103 / 0973-029X.174614. PMID: 26980961 ; PMCID: PMC4774286.

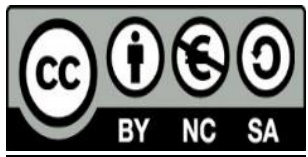

